\title{
Redes neurais artificiais aplicadas ao processo de coagulação
}

\author{
Artificial neural networks applied to the coagulation process
}

\author{
Fábio Conceição de Menezes \\ Engenheiro Ambiental pela Faculdade de Tecnologia e Ciências de Salvador (FTC) \\ Karla Patricia Santos Oliveira Rodriguez Esquerre \\ Pesquisadora da Rede de Tecnologias Limpas (Teclim) da Universidade Federal da Bahia (UFBA) \\ Ricardo de Araújo Kalid \\ Professor do Departamento de Engenharia Química da UFBA \\ Asher Kiperstok \\ Coordenador da Rede Teclim e do Programa de Pós-graduação em Produção Limpa da UFBA \\ Mario Cezar de Oliva Matos \\ Pesquisador da Rede Teclim da UFBA \\ Rodrigo Moreira \\ Engenheiro da Unidade de Insumos Básicos da Braskem
}

\section{Resumo}

A coagulação é uma etapa de tratamento da água, e para tal são realizados ensaios de teste de jarro que permitem determinar a dose necessária dos agentes coagulante e químico de ajuste de pH no processo de coagulação. Contudo, esses ensaios demoram a ser executados, não respondendo em tempo real às mudanças da qualidade da água bruta. Para superar tal limitação, redes neurais artificiais multicamadas foram construídas (e seus pesos sinápticos ajustados), validadas e testadas para predizer a dosagem do hidróxido de sódio e do sulfato de alumínio - utilizados como agentes químico de ajuste de pH e coagulante, respectivamente. Os resultados dos modelos obtidos são compatíveis com os dados experimentais tendo em vista que as incertezas das estimativas estão na mesma ordem de grandeza das faixas indicadas pelos ensaios realizados de testes de jarro ao longo de quase seis anos.

Palavras-chave: redes neurais artificiais; coagulação; teste de jarro; sulfato de alumínio; hidróxido de sódio.

\section{Abstract}

Coagulation is a stage in water treatment and, for this, jar tests are performed, which allows determining the optimal coagulant and alkalizer doses in coagulation process. However, these tests are time-consuming and do not enable real-time responses to changes in raw water quality. To overcome these limitations, artificial multilayer perceptron neural networks were built, trained, validated and tested to predict the aluminum and sodium hydroxide doses - used as coagulant and alkalizer, respectively. The results of these models are encouraging to consider that the estimated uncertainties have the same order of the variation limits magnitude indicated by the jar tests for almost a six-year period.

Keywords: artificial neural networks; coagulation process; jar test; alum; sodium hydroxide. 


\section{Introdução}

O Polo Industrial de Camaçari, localizado a 45 quilômetros de Salvador, demanda aproximadamente $5.700 \mathrm{~m}^{3} / \mathrm{h}$ de água para produzir mais de oito milhões de toneladas de produtos químicos e petroquímicos por ano. Cerca de 90\% dessa água é fornecida pela Unidade de Insumos Básicos da Braskem (UNIB), após processamento em sua Unidade de Tratamento de Água (UTA). Nesta unidade, há a produção de água clarificada, filtrada, desmineralizada e potável. O processo de clarificação compreende as seguintes operações unitárias: coagulação, floculação e sedimentação.

No processo de coagulação realizado na UTA, o agente coagulante utilizado é o sulfato de alumínio $\left(\mathrm{Al}_{2}\left(\mathrm{SO}_{4}\right)_{3} \cdot \mathrm{nH}_{2} \mathrm{O}\right)$, e o agente químico de ajuste do $\mathrm{pH}$ é o hidróxido de sódio $(\mathrm{NaOH})$. O primeiro tem por função reduzir as forças que tendem a manter separadas as partículas em suspensão, enquanto que o $\mathrm{NaOH}$ mantém o pH da solução durante o processo de coagulação dentro de uma faixa desejável para que a ação do coagulante seja satisfatória. A dosagem de ambos agentes depende da qualidade da água a ser tratada e é obtida por meio de um ensaio denominado teste de jarro.

Assim como ocorre com a maior parte das águas superficiais naturais, a água do rio Joanes, que abastece a UTA, caracteriza-se por amplas variações de qualidade (MENEZES, 2008). Dessa forma, o tempo necessário para realização do teste e, especialmente, as baixas frequências da sua realização, não permitem respostas em tempo real às mudanças na qualidade da água a ser tratada (MAIER et al, 2004).

O objetivo principal deste trabalho é desenvolver um modelo que forneça predições das dosagens do agente químico de ajuste do $\mathrm{pH}$ e de agente coagulante na UTA. Para tanto, modelos de redes neurais artificiais (RNAs) foram construídos, validados e testados, fazendo-se uso dos resultados dos testes de jarro e das medidas de qualidade de água tratada, e considerando uma série histórica de quase seis anos.

O modelo desenvolvido a partir de RNAs perceptron de múltiplas camadas forneceu melhores resultados de predição em comparação aos modelos construídos a partir de regressão linear múltipla com os mesmos dados (MENEZES, 2008), devido a não-linearidade das inter-relações das variáveis que estão envolvidas no processo de coágulo-floculação e que podem ser captadas pelas RNAs. As RNAs são sistemas computacionais estruturados pelo processo de aprendizagem a partir da apresentação de dados. Os modelos gerados são do tipo "caixa-preta", em que um aproximador de função busca a generalização do sistema que lhe foi apresentado promovendo um ajuste de seus pesos sinápticos, o que lhe confere a habilidade de detecção e mimetização de estruturas.

\section{Metodologia}

\section{Descrição dos dados}

O teste de jarro é realizado para simular o processo de coagulação, floculação e sedimentação em unidades de tratamento de água. Em resumo, nesse ensaio amostras da água a serem tratadas são coletadas e colocadas em um equipamento provido de agitadores; daí diferentes dosagens do agente coagulante são aplicadas numa faixa de $\mathrm{pH}$ regulada pela adição de um agente químico regulador do $\mathrm{pH}$ (no caso da UTA, um alcalinizante). Observam-se, então, os seguintes parâmetros para determinar qual a melhor dosagem testada: tempo e gradiente de velocidade de mistura rápida; tempo e gradiente de velocidade de floculação; e velocidade de sedimentação.

Dados de qualidade da água bruta e decantada - com os respectivos resultados dos ensaios do teste de jarro - foram fornecidos pela empresa para a construção do modelo. Assim, como variáveis preditoras, utilizou-se cor, sólidos suspensos (SS), turbidez, pH e alcalinidade, e, como variáveis preditas, as dosagens de sulfato de alumínio e de hidróxido de sódio, ambos utilizados no processo de modelagem. Os dados referentes à dosagem ótima dos resultados dos testes de jarro foram selecionados buscando os valores de menor dosagem que produziram uma água tratada com os parâmetros de qualidade (SS, cor, turbidez e alumínio residual) dentro dos limites impostos para especificação de água clarificada e numa condição operacional desejável, totalizando 310 dados após exclusão de valores considerados aberrantes para os especialistas do processo. Os gráficos de séries temporais das variáveis e suas estatísticas básicas são apresentados na Figura 1 e Tabela 1, respectivamente. Juntamente com as estatísticas básicas, foi realizado o teste de normalidade de Kolmogorov-Smirnov das variáveis para um intervalo de confiança de $95 \%$ obtendo o valor de $\mathrm{p}<0,005$.

Os padrões de comportamento cíclico estão relacionados às variações sazonais da região, ou seja, com exceção da alcalinidade, verifica-se que em períodos chuvosos os parâmetros de qualidade da água apresentam picos possivelmente ocasionados pelo arraste de materiais para o rio e pelo revolvimento de suas águas. $\mathrm{O} \mathrm{pH}$ da água do rio Joanes apresenta um decaimento gradual no decorrer dos seis anos, comportamento ainda não explicado. Os gráficos indicam ainda a presença de valores atípicos (picos), que foram investigados através de um estudo multivariado pela técnica de análise de componentes principais (PCA), descrita a seguir.

\section{Tratamento e divisão de dados}

Além do procedimento de seleção de dados citado anteriormente, a técnica de análise de componentes principais foi utilizada na investigação de valores destoantes do comportamento amostral e da associação das variáveis. Através do gráfico dos scores das variáveis preditoras 

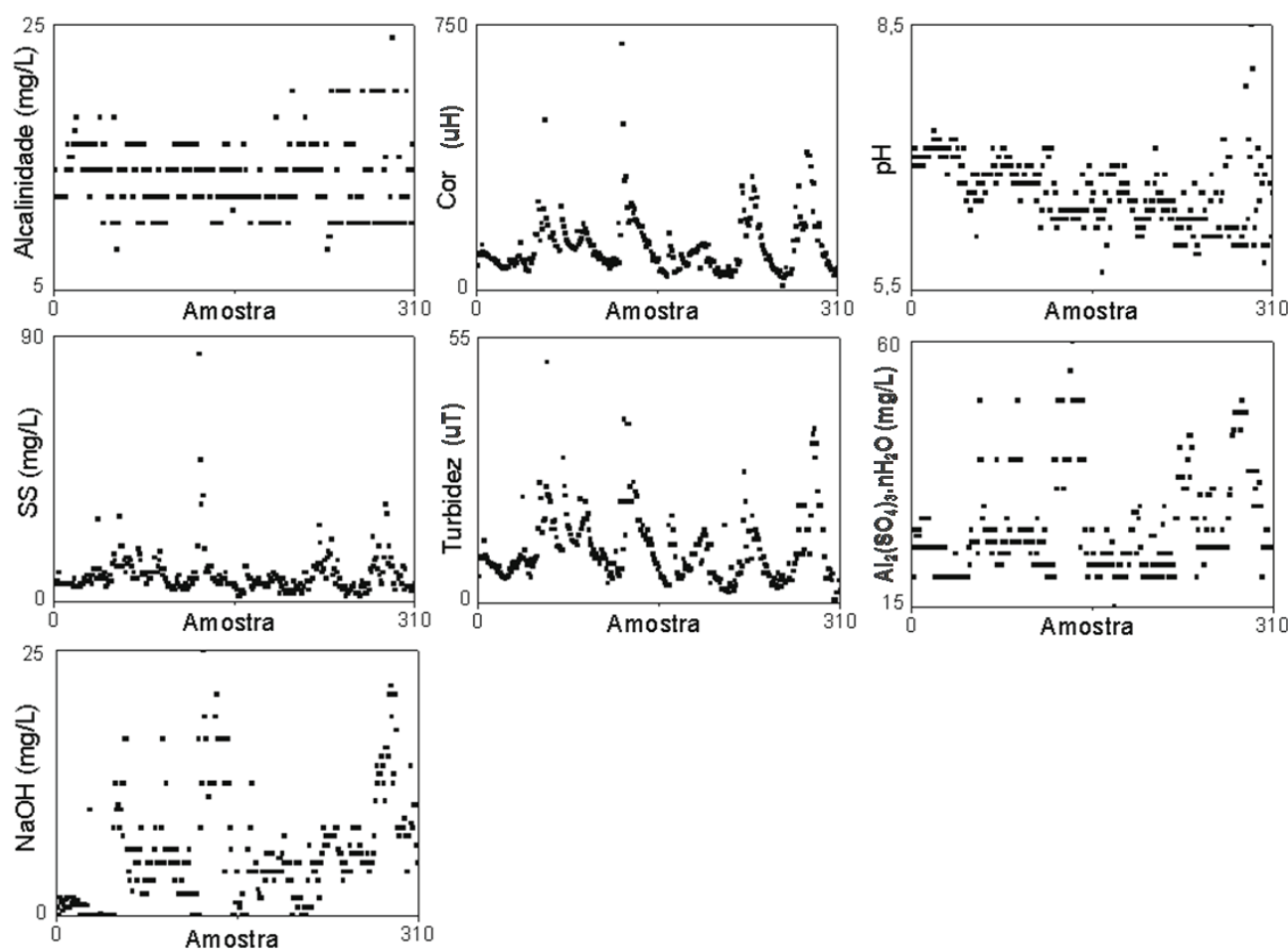

Figura 1 - Séries temporais das variáveis preditoras e preditas para um período de seis anos (janeiro de 2000 a dezembro de 2006)

(Figura 2A), verifica-se a presença de uma observação afastada da massa de dados, indicando-se como um possível valor aberrante. Tal observação foi excluída do conjunto de dados utilizado. Já a formação de agrupamentos no gráfico dos loadings (Figura 2B) indica a presença de associação entre variáveis que apresentam certa dependência física e cujos coeficientes de correlação são significativos (Quadro 1).

Após organização dos dados de forma aleatória, os 309 dados foram divididos em três subconjuntos: (i) conjunto de treinamento para ajuste dos pesos das conexões; (ii) conjunto de validação para determinar em que momento parar o treinamento e otimizar a arquitetura da rede e os parâmetros internos do modelo (por exemplo, taxa de aprendizagem e momentum); e (iii) conjunto de testes para testar a habilidade de generalização do modelo para um novo conjunto de dados. O tratamento de dados foi realizado utilizando-se o pacote comercial Minitab (versão 15.0).

\section{Construção dos modelos}

A arquitetura da RNA se constitui de uma perceptron multicamadas (MLP), pois tem sido usada com sucesso na predição de doses de agentes coagulantes (BAXTER et al, 1999; Maier et al, 2004) e em outros tipos de variáveis hídricas e ambientais (MAIER; DANDY, 2000; OLIVEIRA-ESQUERRE et al, 2004).

O número de camadas intermediárias e neurônios intermediários foram estimados por tentativa e erro. Apesar de já ter sido comprovado que uma rede neural com uma camada intermediária pode
Tabela 1 - Estatísticas básicas das variáveis preditoras e preditas

\begin{tabular}{lccccc} 
Variáveis & Unidade & Média & DP & Mínimo & Máximo \\
\hline $\mathrm{Al}_{2}\left(\mathrm{SO}_{4}\right)_{3} \cdot \mathrm{nH}_{2} \mathrm{O}$ & $\mathrm{mg} / \mathrm{L}$ & 28,8 & 8,8 & 15 & 60 \\
$\mathrm{NaOH}$ & $\mathrm{mg} / \mathrm{L}$ & 7,1 & 6,1 & 0 & 30 \\
\hline Alcalinidade & $\mathrm{mg} / \mathrm{L}$ & 13,5 & 2,8 & 8 & 24 \\
$\mathrm{Cor}$ & $\mathrm{uH}$ & 125,1 & 81,2 & 11 & 696 \\
$\mathrm{pH}$ & - & 6,6 & 0,4 & 5,7 & 8,5 \\
$\mathrm{SS}$ & $\mathrm{mg} / \mathrm{L}$ & 9 & 7,2 & 2 & 84 \\
Turbidez & $\mathrm{uT}$ & 11,1 & 7,2 & 0,5 & 49,9
\end{tabular}

*Teste de normalidade de Kolmogorov-Smirnov para um intervalo de confiança de 95\% (valor de $p>0,05$ ).

aproximar qualquer função contínua, dado suficiente grau de liberdade (HORNIK et al, 1989), outros estudos demonstram que uma rede com mais de uma camada pode fornecer maior flexibilidade e permitir aproximações de funções complexas com menos conexões (FLOOD; KARTAM,1994). Dessa forma, buscou-se utilizar no máximo duas camadas intermediárias.

O número de neurônios intermediários também foi encontrado por tentativa e erro. Maier et al (2004) sugerem que o número máximo de neurônios intermediários seja de $2 I+1$, onde I é o número de neurônios de entrada. Já Nascimento et al (2000) atentam para o fato de que o número de pesos a serem ajustados é maior que o número de dados de entrada, e que é possível que venha a ocorrer o chamado sobreajuste, no qual a rede representa muito bem os dados usados no treinamento (ajuste de peso da RNA), mas perde a capacidade de generalizar para outras situações. Neste trabalho, o número máximo 
Quadro 1 - Matriz de correlação.

\begin{tabular}{|c|c|c|c|c|c|c|}
\hline & $\mathrm{pH}$ & Cor & sS & Turbidez & Alcalinidade & $\mathrm{NaOH}$ \\
\hline $\mathrm{pH}$ & 1 & & & & & \\
\hline Cor & $-0,191$ & 1 & & & & \\
\hline SS & $-0,11$ & 0,761 & 1 & & & \\
\hline Turbidez & $-0,014$ & 0,822 & 0,648 & 1 & & \\
\hline Alcalinidade & 0,074 & $-0,151$ & $-0,04$ & $-0,141$ & 1 & \\
\hline $\mathrm{NaOH}$ & $-0,292$ & 0,683 & 0,487 & 0,583 & $-0,133$ & 1 \\
\hline $\mathrm{Al}_{2}\left(\mathrm{SO}_{4}\right)_{3} \cdot \mathrm{nH}_{2} \mathrm{O}$ & $-0,274$ & 0,725 & 0,481 & 0,573 & $-0,096$ & 0,859 \\
\hline
\end{tabular}

Em negrito estão os coeficientes de correlação com significância estatística para um intervalo de confiança de $95 \%$ (ou seja, valor de $p<0,05$ ).

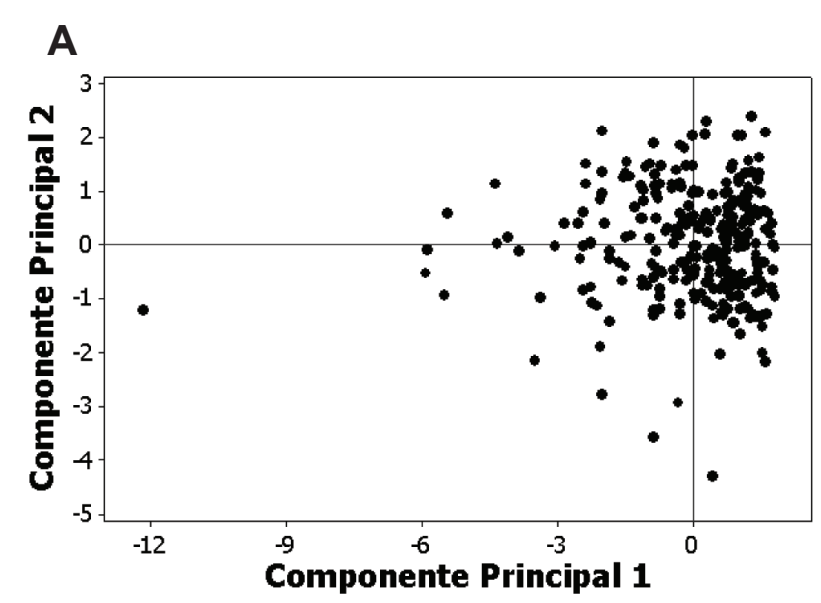

B

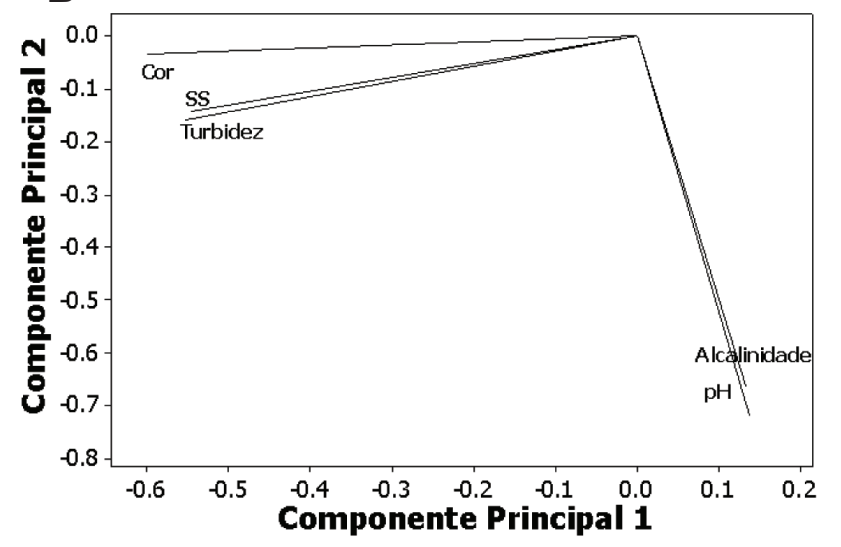

Figura 2 - Gráficos de scores (A) e loadings (B) correspondentes às componentes principais 1 e 2

de neurônios intermediários utilizados foi 22, em concordância com a sugestão de ambos os autores.

As RNAs foram treinadas usando a versão do algoritmo de retropropagação momentum (HAYKIN, 2001), na tentativa de promover uma aceleração da convergência (JACOB, 1998). Os seus parâmetros de passo e momentum foram encontrados por tentativa e erro; as faixas testadas incluíram de 0,15 a 0,85 para cada parâmetro. Foram testadas as funções sigmoidal e tangente hiperbólica como funções de transferência da camada intermediária.
O critério de parada do treinamento foi baseado no erro quadrático médio do conjunto de validação, e não de treinamento, para garantir a generalização do modelo (OLIVEIRA-ESQUERRE et al, 2004). Já o desempenho relativo dos diversos modelos construídos foi comparado avaliando-se o coeficiente de correlação múltipla $\left(\mathrm{R}^{2}\right)$, o erro absoluto médio (MAE) e o desvio padrão dos resíduos absolutos para os três conjuntos de dados, sendo selecionado o modelo que apresentou melhores resultados para o conjunto de teste. Os modelos foram construídos utilizando-se o pacote comercial Neurosolutions Professional (versão 3.0).

\section{Resultados e discussão}

Na Tabela 2 são apresentados os resultados do modelo que apresentou melhor desempenho, dotado de geometria de duas camadas intermediárias composta por seis neurônios cada.

Os gráficos dos valores medidos versus o preditos do sulfato de alumínio $\left(\mathrm{Al}_{2}\left(\mathrm{SO}_{4}\right)_{3} \cdot \mathrm{nH}_{2} \mathrm{O}\right)$ e do hidróxido de sódio $(\mathrm{NaOH})$ para o conjunto de teste estão dispostos nas Figuras 3A e B, respectivamente, para um intervalo de predição de $95 \%$.

As Figuras 4 e 5 apresentam uma comparação do desempenho da rede neural para o sulfato de alumínio e hidróxido de sódio, respectivamente, considerando o período estudado. Observa-se que os modelos parecem reproduzir parcialmente a variação total das observações no período considerado. Nenhum padrão sistemático é observado no gráfico dos resíduos, e cerca de 68 e 82\% dos resíduos dos modelos de sulfato de alumínio e hidróxido de sódio, respectivamente, apresentaram resíduos abaixo do limite de variação utilizado no processo de clarificação ( $5 \mathrm{mg} / \mathrm{L}$ ).

Este estudo pode ser visto, ainda, como uma análise exploratória das interações existentes entre a qualidade da água e as respectivas dosagens de agente coagulante e alcalinizante, pois o desempenho dos modelos ainda pode ser melhorado. Ressalta-se que o desenvolvimento do estudo permitiu também o conhecimento e descrição dos padrões de variação temporal dos dados de qualidade da água bruta proveniente do Rio Joanes, principal fornecedor de água para as indústrias do polo de Camaçari. 


\section{Conclusões}

O uso de modelos a partir de RNAs permitiu o desenvolvimento de uma metodologia para a construção de uma ferramenta computacional com o intuito de dar suporte ao processo de coagulação. A aplicação dessa metodologia permitirá a construção de modelos que tragam uma economia de tempo e dinheiro, dispondo ao operador uma ferramenta que estime as doses requeridas dos produtos químicos em função das características da água bruta - realizando os ensaios de teste de jarro para acompanhamento e certificação da adequada condição de coagulação pelo que for predito com o modelo, quando assim for necessário.

Os modelos desenvolvidos a partir de RNAs perceptron de múltiplas camadas forneceram resultados de predição compatíveis com os dados experimentais, pelo fato da não-linearidade das inter-relações (das variáveis) poder ser captada pelas RNAs. Contudo, as RNAs não permitem uma abordagem sobre aspectos físicos do sistema, pelo fato de que os pesos sinápticos gerados no processo de modelagem não possuem nenhum significado físico.

Tabela 2 - Resultados do modelo de predição para os conjuntos de treinamento, validação e teste

\begin{tabular}{lcccccccccc} 
& \multicolumn{3}{c}{ Treinamento } & \multicolumn{3}{c}{ Validação } & \multicolumn{3}{c}{ Teste } \\
\cline { 2 - 10 } $\begin{array}{l}\text { Variáveis } \\
\text { preditas }\end{array}$ & $\mathrm{R}^{2}$ & $\mathrm{MAE}$ & $\mathrm{DP}$ & $\mathrm{R}^{2}$ & $\mathrm{MAE}$ & $\mathrm{DP}$ & $\mathrm{R}^{2}$ & $\mathrm{MAE}$ & $\mathrm{DP}$ \\
$\mathrm{Al}_{2}(\mathrm{SO})_{3} \cdot \mathrm{nH}_{2} \mathrm{O}$ & 82 & 2,5 & 2,9 & 76 & 4,3 & 3,6 & 67 & 9,5 & 4,9 \\
$\mathrm{NaOH}$ & 80 & 2 & 1,6 & 74 & 2,9 & 2,5 & 61 & 3 & 2,9
\end{tabular}

$\mathrm{R}^{2}$ : Coeficiência de relação múltipla; MAE: erro absoluto médio; DP: desvio padrão.

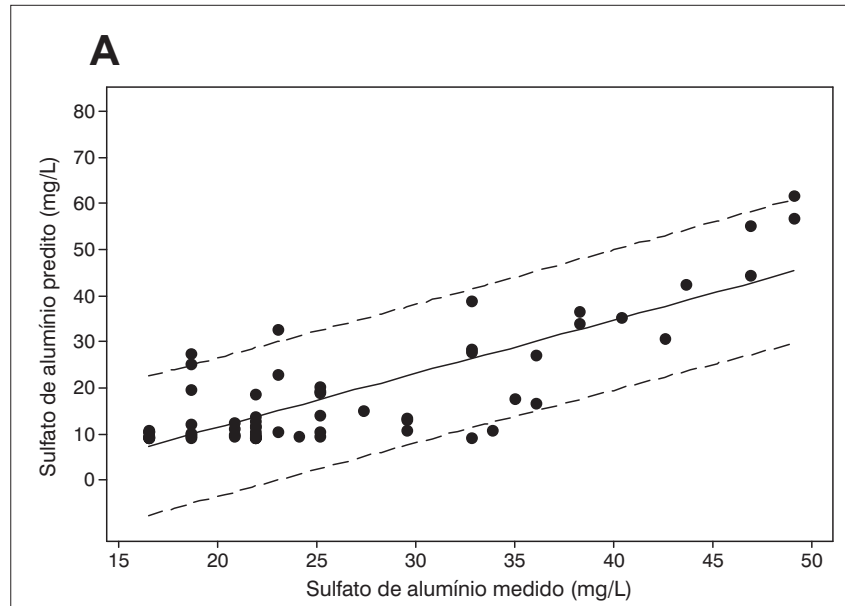

B

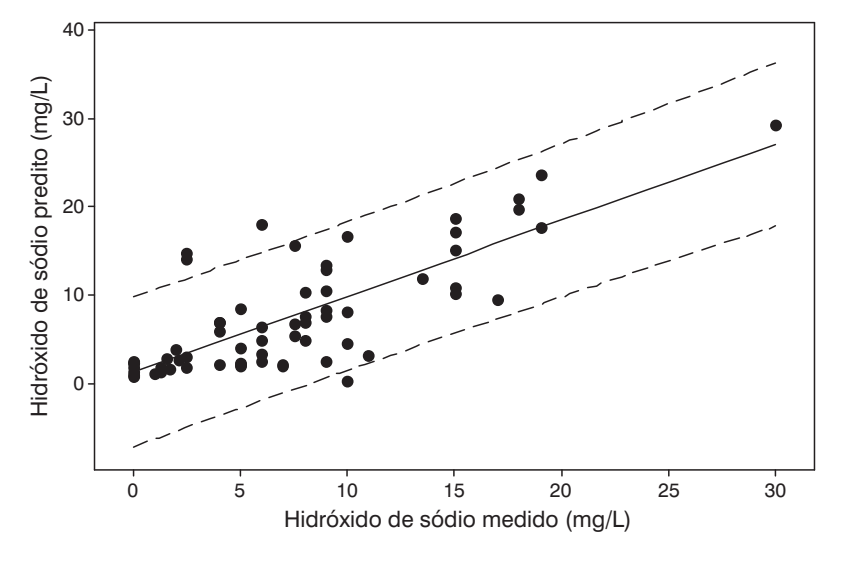

Figura 3 - Relação entre os valores medidos e preditos de (a) $\mathrm{Al}_{2}\left(\mathrm{SO}_{4}\right)_{3}$. $\mathrm{nH}_{2} \mathrm{O}$ e (b) $\mathrm{NaOH}$ (linha contínua) para do conjunto de teste. As linhas tracejadas indicam o intervalo de predição de 95\%
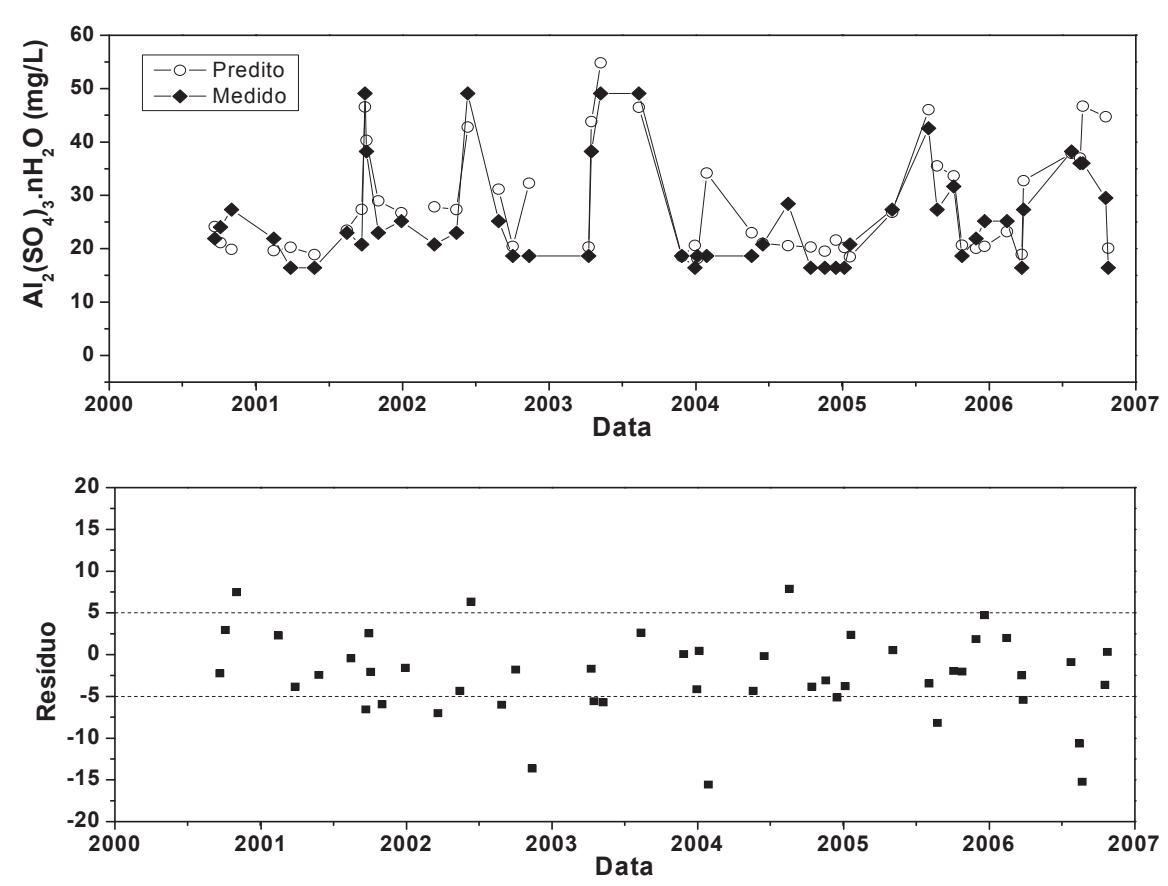

Figura 4 - Séries temporais de valores preditos e medidos de $\mathrm{Al}_{2}\left(\mathrm{SO}_{4}\right)_{3} \cdot \mathrm{nH}_{2} \mathrm{O}$ (superior) e resíduos para o conjunto de teste (inferior) 

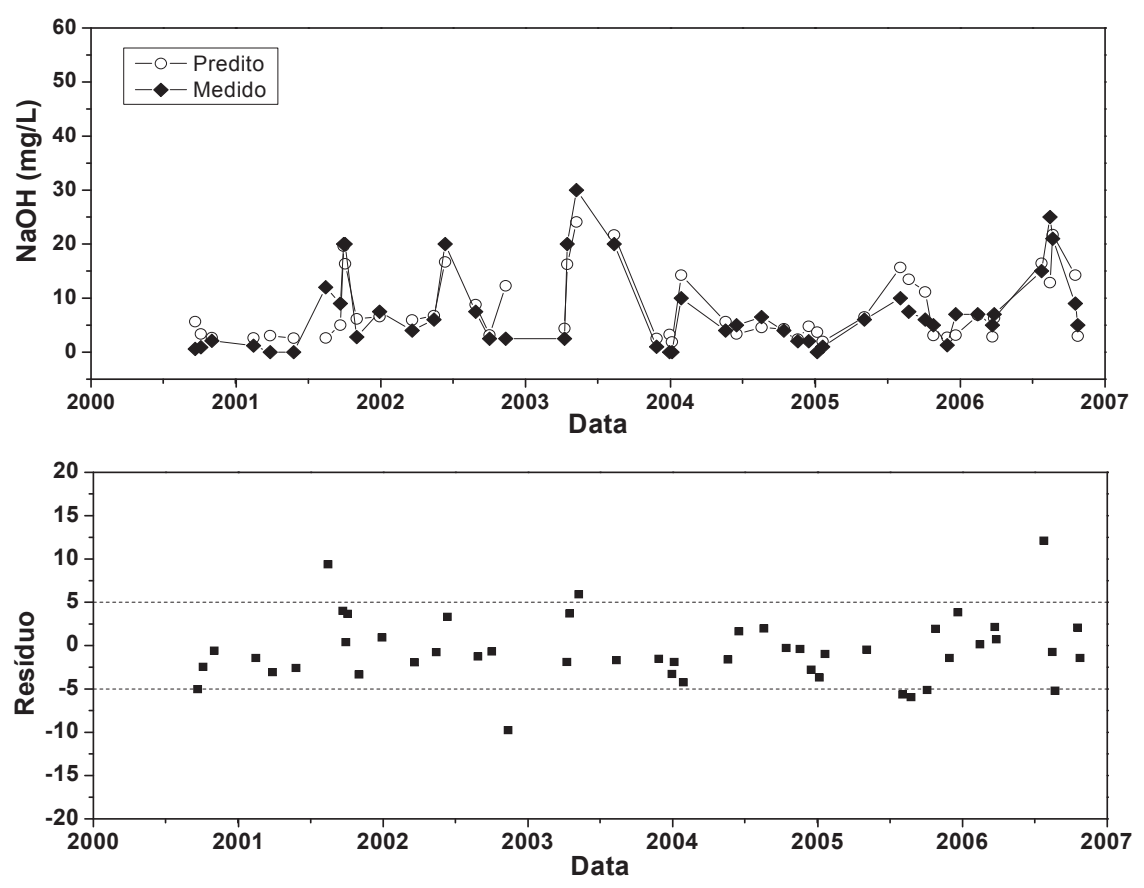

Figura 5 - Séries temporais de valores preditos e medidos de $\mathrm{NaOH}$ (superior) e resíduos para o conjunto de teste (inferior)

Espera-se que esses modelos também sejam usados como uma interface de laboratório virtual, de forma a promover um melhor entendimento dos fatores que afetam a unidade de processo. O próximo passo dessa pesquisa consistirá na busca do aperfeiçoamento dos modelos de predição, fazendo uso da técnica de planejamento de experimentos. Ainda, a variação cíclica percebida nos parâmetros de qualidade da água bruta pode servir de guia para uma futura geração de modelos destinados a distintos períodos do ano.

\section{Referências}

BAXTER, C.W.; STANLEY, S.J.; ZHANG, Q. Development of a full scale artificial neural network model for the removal of natural organic matter by enhanced coagulation. Journal of Water Services Research and Technology-AQUA, v. 48, n. 4, p. 129-136, 1999

FLOOD, I.; KARTAM, N. Neural networks in Civil Engineering. I: principles and understanding. Journal of Computing in Civil Engineering, v. 8, n. 2, p. 131-148. 1994.

HAYKIN, S. Redes neurais: princípios e prática. Tradução: Paulo Martins Engel. 2a. edição. Porto Alegre: Bookman, 2001.

HORNIK, K.; SINCHCOMBE, M.; WHITE, H. Multilayer feedforward networks are universal approximators. Neural Networks, v. 2, n. 5, p. 359-366, 1989

JACOBS, R.A. Increased rates of convergence through learning rate adaptation. Neural Networks, v. 1, n. 4, p. 295-307, 1998.

MAIER, H.R.; DANDY, G.C. Neural networks for the prediction and forecasting of water resources variables: a review of modelling issues and applications. Environmental Modelling \& Software, v. 15, p. 101-124, 2000

MAIER, H.R.; MORGAN, N.; CHOW, C.W.K. Use of neural networks for predicting optimal alum doses and treated water quality parameters. Environmental Modelling \& Software, v.19, p. 485-494, 2004.

MENEZES, F.C. Uso de técnicas estatísticas multivariadas e de redes neurais no processo de clarificação da unidade de tratamento de água da Braskem. Monografia (Trabalho de Conclusão de Curso) - Faculdade de Tecnologia e Ciências, Salvador, Bahia, 2008.

NASCIMENTO, C.A.O.; GIUDICI, R.; GUARDANI, R. Neural network based approach for optimization of industrial chemical processes. Computers and Chemical Engineering, v. 24, n. 9-10, p. 2303-2314, 2000.

OLIVEIRA-ESQUERRE, K.P. et al. Application of steady-state and dynamic modeling for the prediction of BOD for an aerated lagoon at a pulp and paper mill. Part I: linear approaches. Chemical Engineering Journal, v.105, n. 1-3, p. 61-69, 2004. 